\section{The BCS Annual Conference, Manchester, 6-8 June 2016. The Vice-President's message}

\author{
Cliff Garratt
}

The BCS Annual Conference is evolving into a different beast from that which I recall both during my time as a trainee and more recently. During the 1990s, it seemed as though the Conference provided the focus for all cardiology-related activities in the UK, hosting the key individuals and research from all the subspecialties. I think it is fair to say that, with the subsequent emergence of the national subspecialist meetings, the Annual Conference lost its way to a large degree and with it a considerable proportion of its audience. It took a while for the Society to realise that change was needed if we were to host an event that shone out from the crowd. In the last few years, the focus has been on providing education across a broad spectrum of cardiovascular medicine, fulfilling the needs of cardiology trainees and the cardiovascular community more widely. Cardiologists now need a single meeting that can give them a perspective on all aspects of cardiovascular medicine that lie outside the interest of their particular subspecialty and the Annual Conference is the only event to provide this. The 2015 Conference saw a step-change in terms of increased collaboration with the British Heart Foundation, not only in terms of presentation of the highest quality cardiovascular research, but also in regard to the profile of the meeting to the wider public nationally. For the first time that I am aware, the Conference was the subject of editorial pieces in the national press and wider media coverage generally was very evident. The Programme Committee have recognised this trajectory and the 2016 Conference has been designed to push even further in this direction.

\section{THEME OF 'PREDICTION AND PREVENTION'}

This year our theme is 'Prediction and Prevention' and several of the keynote lectures and sessions share this focus. The conference will be opened by the

Correspondence to Professor Cliff Garratt, Manchester Heart Centre, Central Manchester University Foundation Trust, Manchester Royal Infirmary, Manchester M13 9WL, UK; clifford.garratt@cmft.nhs.uk
President, Dr Sarah Clarke, followed by the opening lecture on 'Big data: a big deal for cardiology?' delivered by Professor Victor Mayer-Schonberger. This year's Sir Thomas Lewis lecture will be delivered by Professor Michael Ackerman from the Mayo Clinic (Prediction and prevention of sudden cardiac death in the young). Professor Ackerman (figure 1) is an active clinician studying genomics and genotype-phenotype relationships in heritable cardiovascular diseases and is widely considered as the world's foremost authority in this area. This year's BCS Lecture (Elucidating the genetic basis of coronary artery disease; implications for prediction, prevention and treatment) will be delivered by Professor Sir Nilesh Samani, knighted in the 2015 New Year Honours list for services to medicine and medical research (figure 2).

\section{COLLABORATION WITH THE BRITISH HEART FOUNDATION}

The British Heart Foundation is a key supporter of the Annual Conference and this collaboration has increased significantly for 2016. Professor David Newby will facilitate the BHF 'Bench-to-bedside' session on acute coronary syndromes. The session will consist of four talks on different aspects of the condition, all presented by members of the same unit, encompassing basic, translational and clinical aspects

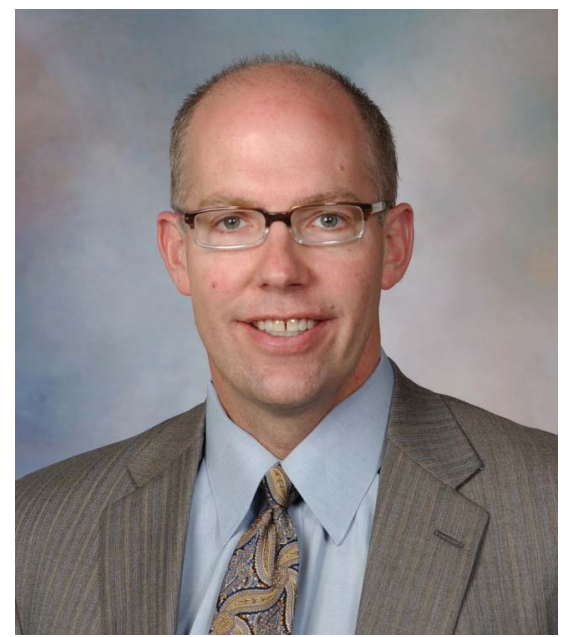

Figure 1 Professor Michael Ackerman

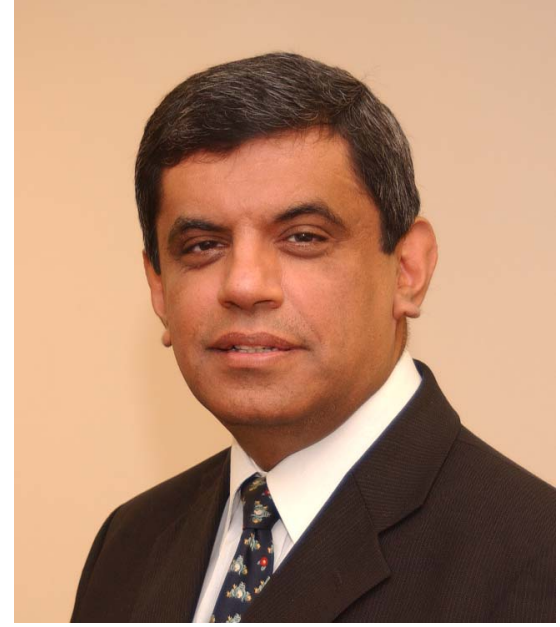

Figure 2 Professor Sir Nilesh Samani

of the disease. The purpose of the session is not only to provide a coordinated, in-depth review of current knowledge of the condition but also to give an insight into the vision and organisation of a successful clinical research department. We think that this aspect will be of particular interest to trainees considering research at some stage in their careers, and for this reason, the Training Day Programme will combine with this session in the Auditorium on the first morning of the Conference.

\section{SCIENTIFIC PROGRAMME \\ HIGHLIGHTS}

Clinical anatomist, author and broadcaster Professor Alice Roberts (figure 3) will discuss what embryology of the heart and other organs has taught us about our evolutionary origins, followed by a talk from Professor Robert Anderson on the relevance of cardiac development to patterns of congenital heart disease.

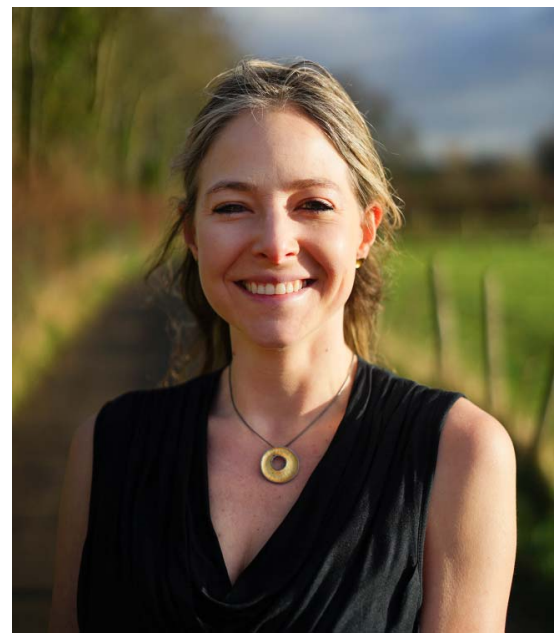

Figure 3 Professor Alice Roberts 


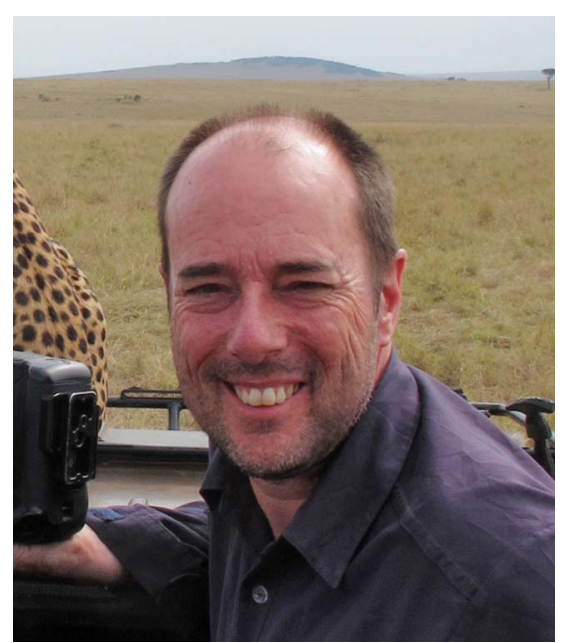

Figure 4 Andy Rouse

Regular attenders at the BCS Annual Conference over the last few years will be aware that we try and deliver 'something completely different' in the Auditorium on the Tuesday afternoon. Last year, it was the very successful 'Music and the Cardiovascular System' and this year it will be 'Photography and the Heart'. In this session, we hope to explore the use of photographic imagery to attract attention and inspire with a multimedia presentation from Andy Rouse (figure 4), one of the most successful wildlife photographers around, followed by short presentations from the finalists in this year's BHF "Reflections of Research" competition.

\section{COLLABORATION WITH THE BRITISH SOCIETY OF CARDIOVASCULAR RESEARCH AND BRITISH ATHEROSCLEROSIS SOCIETY}

The 'basic science track' is provided by the BSCR/BAS Spring meeting, integrated into the first 2 days of the Conference. This part of the meeting is always extremely well attended and of very high quality in terms of scientific content. This year, the meeting is focusing on New Frontiers in Cardiovascular Science with sessions including (1) what is the best way to target inflammation in cardiovascular disease?, (2) exosomes and cardiovascular disease, (3) embryonic programming of cardiovascular disease, (4) non-coding RNAs in basic and translational cardiovascular science. Collaboration between basic and clinical scientists has increased for 2016 with a highlight session on Novel concepts in cholesterol lowering-How to target PCSK9 to improve patient outcomes, organised jointly by BCS, BSCR and BAS, and a new combined Young Investigators Prize run by all the three organisations.

\section{COMPREHENSIVE COVERAGE OF ALL THE NEW DEVELOPMENTS IN CARDIOLOGY AND CARDIOVASCULAR SCIENCE}

- National training day: including SAC session, ECG interpretation with Drs Fynn and Rowland and MDT-Live on cardiac assessment prior to non-cardiac surgery.

- Imaging: dedicated imaging track; abstract poster sessions; integrated imaging content in the highlight sessions of the programme; sessions on pericardial disease, mitral valve disease and investigation of dilated cardiomyopathy.

- Arrhythmias: sessions on AF ablation and on first-line management of cardiac arrhythmias: arrhythmia abstract sessions; discussion of arrhythmia clinical cases; the Sir Thomas Lewis lecture; modern management of syncope; ESC guidelines on ventricular arrhythmias with Karl-Heinz Kuck and John Camm.

- Intervention: BHF bench-to-bedside session on acute coronary syndromes; BCIS sessions on myocardial infarction and percutaneous management of structural heart disease; sessions on refractory angina and assessment of patients with suspected stable angina; BHVS session on intervention in valve disease: abstract sessions; discussion of interventional clinical cases (MDT-Live); a joint session with the British Association of Stroke Physicians on Thrombectomy for acute ischaemic stroke: a new role for the cardiologist?

- Heart failure: sessions on new approaches to heart failure therapy, UK clinical trials in heart failure, acute cardiomyopathies and investigation of dilated cardiomyopathy: cardio-oncology; discussion of heart failure clinical cases (MDT-Live).

- Adult congenital heart disease; a BCCA session on congenital left ventricular outflow tract obstruction; obstetric cardiology; Kawasaki disease; genetic syndromes in ACHD.
- 'Hypertension for cardiologists' featuring the British Hypertension Society with a line-up of international-quality speakers.

- Resuscitation. Highlight session on out-of-hospital cardiac arrest including 10 steps to improving outcomes from out-of-hospital cardiac arrest from Professor Thomas Rea (Seattle, USA) and patient scenarios from the Resuscitation Council UK.

- Education for revalidation (E4R) sessions including 'Top 10 Trials' and 'Life Long Learning' and the ever-popular BCS Quiz hosted by Rod Stables.

- Multisession tracks dedicated to BHF Alliance Healthcare Professionals, BACPR/BANCC/BSH members, Patients (CCPUK) and Healthcare Scientists. Includes keynote lecture on Maximising quality and safety of care for people with heart failure by Professor Sandra Dunbar, Emory University, US.

- International sessions in association with the European Society of Cardiology and the American College of Cardiology.

Finally, I continue to be hugely impressed by the willingness of cardiologists and cardiovascular scientists, both from the UK and internationally, to give time and effort to the planning of this meeting. In particular, I would like to acknowledge the help of the BHF team and the staff, officers and Programme Committee of the BCS. The full programme and booking for lifelong learning, simulation, imaging village, and the annual dinner are available online at http://www.bcs.com/Conference. An App containing the Programme and biographies of the faculty is also available (BCS Conf 2016). I have absolutely no doubt that this year's Conference will be the most enjoyable and educational yet.

\section{Competing interests None declared.}

Provenance and peer review Commissioned; internally peer reviewed.

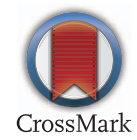

To cite Garratt C. Heart 2016;102:809-810.

Heart 2016;102:809-810.

doi:10.1136/heartjnl-2016-309862 